\title{
MARKOV LIMID PROCESSES FOR REPRESENTING AND SOLVING RENEWAL PROBLEMS
}

\author{
D. NILSSON
}

Department of Animal Science and Animal Health, Royal Veterinary and Agricultural University and Aalborg University, Aalborg, Denmark

E-mail: deni@nykredit.dk

\author{
A.R. KRISTENSEN \\ Department of Animal Science and Animal Health, Royal Veterinary and Agricultural \\ University, Grønnegårdsvej 3, DK-1870 Frederiksberg C, Copenhagen, Denmark \\ E-mail:ark@dina.kvl.dk
}

\begin{abstract}
In this paper a new tool for simultaneous optimization of decisions on multiple time scales is discussed. It combines the dynamic properties of Markov decision processes with the flexible and compact state space representation of Limited Memory Influence Diagrams (LIMIDs).
\end{abstract}

\section{INTRODUCTION}

In this paper we discuss a class of management problems that are characterized by events taking place at two levels. At the child level some kind of agent is performing a sequence of actions that are of interest to a decision maker. At the founder level the agent is reset, renewed or replaced either as an inherit aspect of the problem or as the consequence of a decision made by the decision maker. There are several management problems that fit into such a framework. Among the more obvious examples we shall mention optimal maintenance and replacement of assets (like machines) where the renewal is expressed as the replacement of the old asset by a corresponding new one. An other example is robot navigation where the renewal event could be entrance of an other room whereas the navigation within the same room involves a sequence of actions aiming at reaching the other room.

Inspired by the maintenance and replacement problem in livestock production Kristensen (1988) introduced the notion of hierarchical semi-Markov decision processes where the stages of a founder level process correspond to assets successively replacing each other in an infinite chain of assets. Transition to a new stage at the founder level corresponds to a replacement. Furthermore each individual asset is modeled at the child level by a separate finite time semi Markov decision process representing the states and decisions corresponding to daily maintenance of the asset. In livestock models very large models (Houben et al., 1994; Verstegen et al., 1998; Mourits et al., 1999) have been developed using the hierarchical technique which has had a 
tremendous effect on efficiency of optimization. Later the concept was further developed by Kristensen and Jørgensen (2000) into multi-level hierarchical Markov processes in order to allow for simultaneous optimization of decisions on multiple time scales (at the founder level as well as at the child levels).

Despite the numerous successful applications, the very concept of a semi Markov decision process has some built-in limitations:

State space representation Most often the state of the system (e.g. an animal) being modeled is defined by the values of a number of state variables each representing a trait of the system. The state space is then defined as the cartesian product of all value sets of individual state variables. This leads to very large transition matrices which - even though they are often sparse - are inefficient from a numerical point of view.

Observability It is generally assumed that the state space of a Markov decision process is fully observable. In particular when we are dealing with production assets, it would be more obvious to distinguish between directly observable state variables like for instance number of items produced and an underlying unobservable production potential of the asset in question. This kind of modeling is referred to as POMDP Partially Observable Markov Decision Processes. Refer for instance to Lovejoy (1991) for a survey or Kaelbling et al. (1998) for an introduction. Unfortunately, POMDPs are intractable even for modest size decision problems.

Markov property The Markov assumption implies that the state at any stage of the problem captures all relevant information about the system. In a straight forward formulation of a decision problem this is rarely the case. The trick used most often in order to make the process Markovian is to include memory variables in the state space. Even though this solves the problem from a theoretical point of view it contributes significantly to the curse of dimensionality of such models.

Even though the multi-level hierarchical Markov decision processes to some extent compensate for these problems by partitioning the state space according to temporal considerations, and Kristensen (1993) has presented a framework to combine hierarchic Markov processes with Bayesian updating in special cases, the state space representation, the lacking observability and the Markov property remain important limitations for the use of semi Markov decision processes.

Influence diagrams (Howard and Matheson, 1984; Shachter, 1986; Smith, 1989; Oliver and Smith, 1990; Jensen, 2001) at first glance seem to overcome all three problems: Instead of a state space formed as a cartesian product, the state variables are represented one by one; variables may be observable or unobservable; and "no forgetting" is implicitly assumed. In practice, however, the performance of influence diagrams has been disappointing. Even though algorithms for optimization are available (refer for instance to Cowell et al. (1999), Chap. 8) the numerical calculations become prohibitive for real world decision problems because of the "no forgetting" assumption. A recent extension by Lauritzen and Nilsson (2001) to influence diagrams called LIMIDs or LImited Memory Influence Diagrams relaxes the "no forgetting" assumption and provides a computationally tractable decision problem without assuming a Markov process. The algorithm presented is exact in some cases and only approximate in other cases. 


\begin{tabular}{|c|c|c|c|}
\hline$(1,1)$ & $(1,2)$ & $(1,3)$ & $\begin{array}{c}(1,4) \\
\text { bat }\end{array}$ \\
\hline$(2,1)$ & $(2,2)$ & $(2,3)$ & $(2,4)$ \\
\hline$(3,1)$ & $(3,2)$ & $(3,3)$ & $(3,4)$ \\
\hline $\begin{array}{r}(4,1) \\
\text { goal }\end{array}$ & $(4,2)$ & $(4,3)$ & $(4,4)$ \\
\hline
\end{tabular}

Figure 1: A robot navigation problem

An other problem of influence diagrams as well as LIMIDs is the static nature of such models. Even though decisions are ordered according to time so that a kind of multi-stage decision problem is handled, time is not an integrated element of the method. It is implicitly assumed that there is no time preference concerning the rewards (or utilities as they are denoted in influence diagrams). Furthermore each time stage has to be explicitly defined in the model as opposed to Markov decision processes. As a consequence, influence diagrams and LIMIDs cannot handle infinite horizon problems. The method is therefore not suitable for the dynamic decision problems in herd management.

The purpose of this paper is to discuss the combination of multi-level Markov processes with LIMIDs in order to provide a tool to integrate the compact and efficient state space representation of LIMIDs allowing for unobservable variables with the dynamic nature of semi Markov decision processes. This is done by defining a semi Markov decision process at the founder level interacting with slightly modified LIMIDs at the child level. We shall illustrate the potential of the combined method through a robot example.

\section{A ROBOT EXAMPLE}

\subsection{Description}

As a simple illustration, consider the robot navigation problem, a gridworld environment as shown in Fig.1. The cells of the $4 \times 4$ grid corresponds to the possible locations of the robot. Two locations have a special meaning: Location $(1,4)$, denoted bat, and connoting battery supply, and location $(4,1)$, denoted goal, and connoting a goal state.

From any location, the robot can perform one of four actions, north (n), south(s), east(e), or west $(w)$, which have a stochastic effect. With probability 0.9 , the actions causes the robot to move one cell in the corresponding direction, and with probability 0.1 the agent moves in the opposite direction. In either case, if the movement would take the robot into a wall, the robot remains in the same cell.

Initially, the robot is loaded with 10 battery units. One single movement in the square requires one battery unit. In location bat, the robot has the option of buying batteri units. It can either buy zero battery units and this action is priceless, buy 5 units at the cost of $5 \$$, or buy 10 units at the cost of of $10 \$$. However, the robot can as a maximum contain 10 batteri units. 
There are also some reward/cost associated with different situations. If the robot is located with zero battery units outside location bat it returns immediately to bat at a cost of $\$ 100$. On the other hand, if the robot reaches location goal, then it immediately receives $\$ 100$.

Every reward/cost is discounted with a discount factor of $\left.\lambda^{t} \in\right] 0,1[$, where $t$ is the number of movements of the robot. The optimization problem consists of maximizing the total discounted reward :

$$
\max _{q} \sum_{t} \mathbf{E}_{q}\left([r(t)-c(t)] \lambda^{t}\right),
$$

where the maximum is over the possible strategies $q$ and $\mathbf{E}_{q}$ is the expectation induced by strategy $q$. The symbols $r(t)$ and $c(t)$ denote the rewards and costs, respectively, at time $t$.

\subsection{Full Observability of Location}

In this version, the robot has perfect knowledge of its present location and it can therefore be represented as a Markov Decision Process with statespace $S$ and acton space $D$, where

$$
\begin{aligned}
S & =\{(1,1),(1,2), \ldots,(4,4)\} \times\{0,1, \ldots, 10\} \\
D & =\{n, e, s, w\} \times\{0,5,10\} .
\end{aligned}
$$

So, the cardinality of $S$ is 176 , and the number of possible actions is 12 , though in every location except bat there are only 4 possible actions.

Despite the small size of the problem, the transition matrix $P\left(s_{t} \mid s_{t-1}, d_{t-1}\right)$ for the location after the $t$ th movement given the previous location and the action at time $t-1$ has no less than $176^{2} \cdot 12>371.000$ entries. Clearly, more realistic versions of the problem may have millions of entries. This curse of dimensionality hinders exact computation of MDPs of even modest size. If, however, a hierarchic process is used, the decision to buy 0,5 or 10 battery units may be modeled at the founder level, and the movement decisions at a child level. The state space is in this way decomposed so that much bigger models become tractable.

In Fig. 2, the problem is shown as a Markov LIMID Process. The problem can be divided into two levels: The decisions at the child level consists of the choice of movements of the robot in between successive visits to the battery location and they are represented by a LIMID. In fact, the LIMID is equivalent to the influence diagram representation. At the founder level there is the "buy" decision.

A throughout description of LIMIDs is given by Lauritzen and Nilsson (2001).

\subsection{Partial Observability of Location}

In this version, the robot does not know its eact location except if it is located in bat. In stead it has two sensors: a north sensor (ns) and a south sensor ( $s s)$. The sensors detect a wall with probability 0.95 in the corresponding directions if there is a wall, and with probability 0.05 detects a wall even though there is no wall in the corresponding direction. 


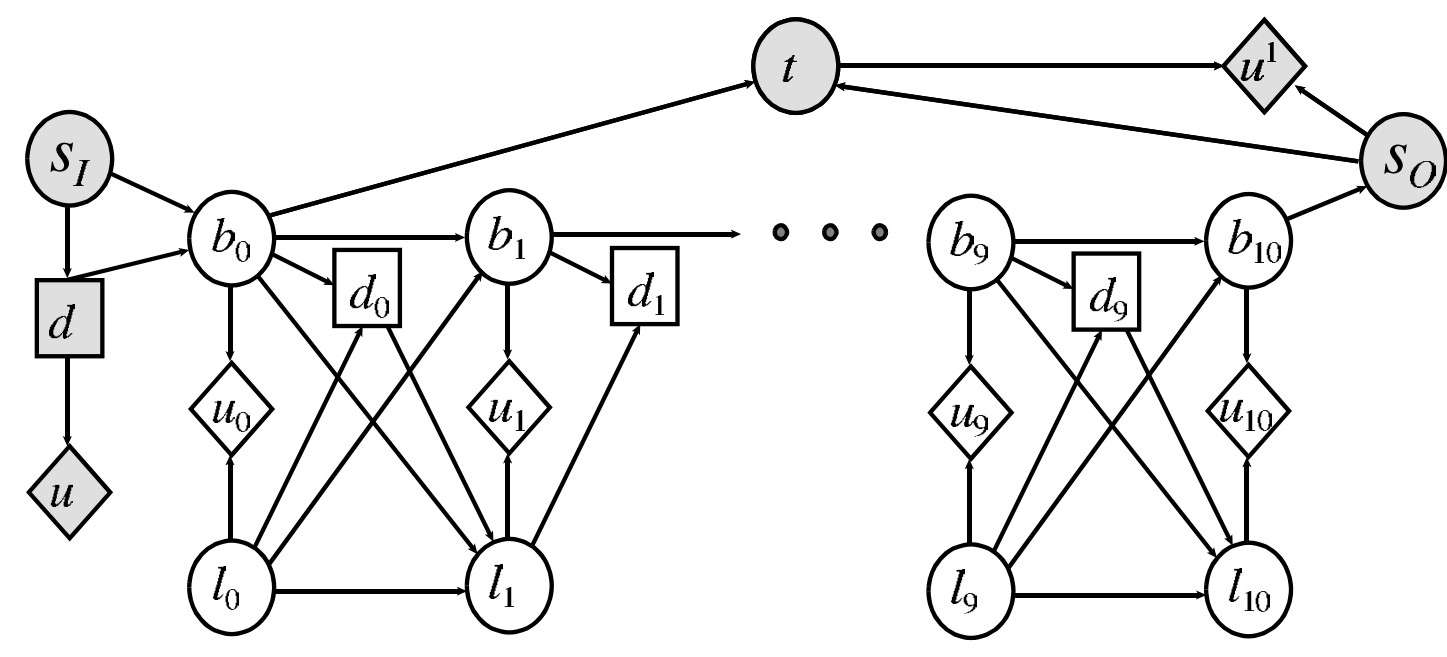

Figure 2: The robot navigation problem with full observability represented as a Markov LIMID Process. Circles are random state variables, squares are decisions and diamonds are utilities. Shaded nodes belong to the founder process $\left(s_{I}, s_{O}\right.$ : the battery level at start and finish; $d$ : the buy decision; $u$ : the costs of the battery units; $t$ : the number of movements in between two successive visits at location "bat"; $u^{1}$ : a node that plays a special role in the solution procedure of the problem as discussed in Section 2.4.). White nodes belong to the child process ( $b_{n}$ : battery level at stage $n ; l_{n}$ : location; $d_{n}$ : move decision; $u_{n}$ : reward) which may be a Markov decision process, an influence diagram or a LIMID. Decisions are made on basis of full knowledge on the location $l_{n}$ and the battery level $b_{n}$.

This version is considerably more challenging than the version with full observability. It corresponds to a Partially Observable Markov Decision Process (POMDP) with state-space $S$ as above. The observation-space $O$ is partly the number of battery units and partly the observations received from the sensors:

$$
O=\{n s, s s\} \times\{0,1, \ldots, 10\}
$$

Unfortunately, POMDPs are highly intractable even for applications of modest size. In Fig. 3, the problem is shown as a modified Markov LIMID Process. Here, the decisions are based on the battery level and the uncertain sensor observations $n s_{n}$ and $s s_{n}$ from the north and south sensor, respectively.

In principle, the decision problem at the child level of Fig. 3 may be solved as an influence diagram, but when the robot has to decide on a movement, all previous sensor inputs and all previous movements from the last visit at bat may affect the optimal choice of movement. As a consequence, a straightforward "influence diagram solution" becomes intractable even for modest size versions of the problem.

By modeling at the child level as a LIMID, we have the option of only considering a subset of the requisite information when making the choice of movement. Clearly, the price we have to pay for disregarding requisite information is that we perform suboptimal actions.

Fig. 3 shows a LIMID where only the present sensor inputs are used for deciding the choice of movements. In particular, we disregard all previous movements and all previous sensor inputs. 


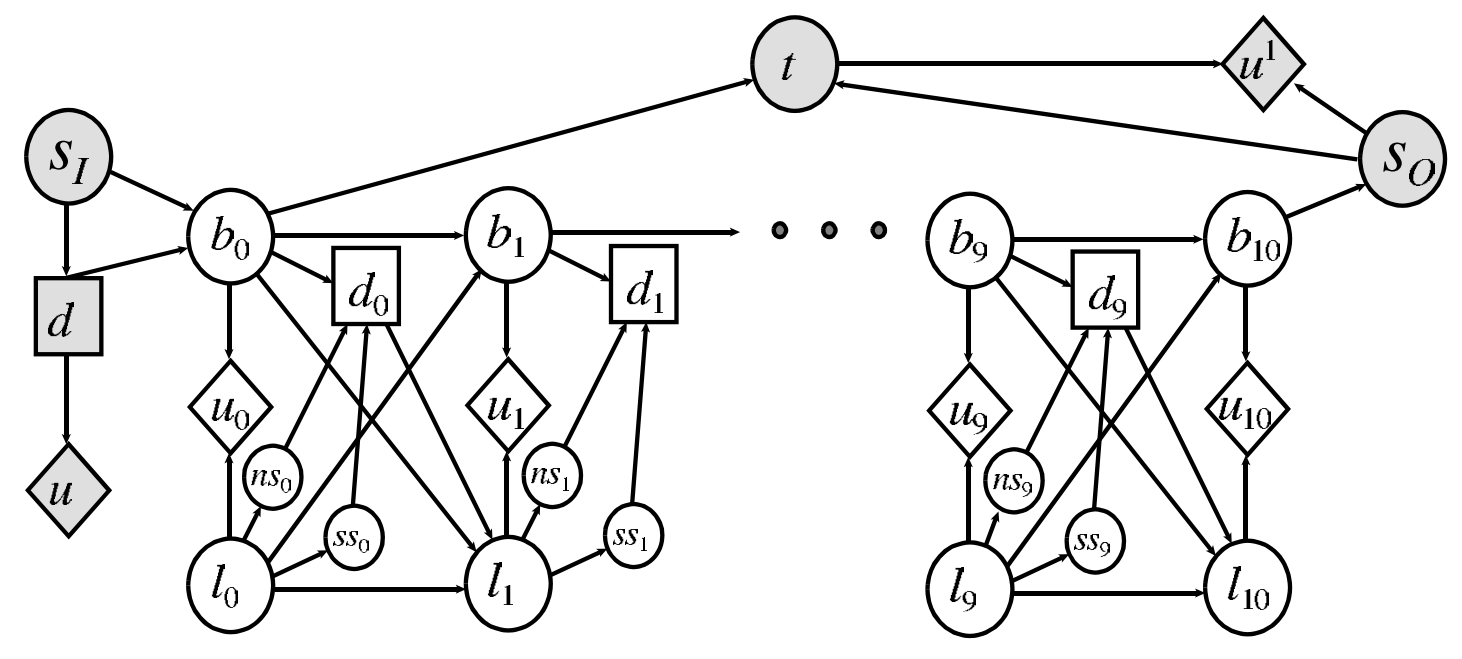

Figure 3: The robot navigation problem with partial observability. Decisions are made on basis of full knowledge on the battery level $b_{n}$, but only uncertain sensor information $\left(n s_{n}\right.$ and $\left.s s_{n}\right)$ about the location.

The intuition is that present sensor inputs, though noisy, provide the robot with more valuable information than past sensor inputs and past actions.

\subsection{Solution of the problem}

The idea behind the algorithm is to establish a protocol for communication between the Markov decision process acting as the founder and the LIMID acting as the child. This is done by defining an input node and and output node in the LIMID. These nodes represent the state of the founder on entry and exit, respectively, of the LIMID. In Fig. 2 and 3 the input and output nodes are denoted $s_{I}$ and $s_{O}$. They are used as basis for calculation of the founder transition probabilities through local calculations in the LIMID.

To solve the problem we apply an iterative procedure that resembles value iteration for a Markov Decision Problem. Our procedure starts with the LIMID $\mathcal{L}^{0}$ in Fig. 3, where $u^{1}\left(s_{0}, t\right)=$ 0 . Next, the following steps are performed until convergence:

1. Compute $v^{n+1}(s)$, the maximal expected utility in $\mathcal{L}^{n}$ given $s_{I}=s$.

2. $\mathcal{L}^{n+1}$ is obtained by letting $u^{1}\left(s_{o}, t\right)=v^{n+1}(s) \lambda^{t}$.

\section{DISCUSSION}

The robot example illustrates several of the desired features. By use of a LIMID rather than a Markov decision process at the child level we obtain a decomposition of the state space as shown in Fig. 2 and 3, where the state variables $b_{n}$ and $l_{n}$ are represented individually instead of by forming the cartesian product of the value sets of the individual variables. Such a separation would be very relevant in the child process of the grassland renewal model presented by Kristensen and Lien (2002). 
It also illustrates the use of unobservable variables as the true location of the robot in Fig. 3. For real-world examples of relevant unobservable state variables, reference is made to the production potential of sows and fields, respectively, in the models presented by Søllested and Kristensen (2002) and Kristensen and Lien (2002).

Furthermore, the LIMID representation at the child level gives us the option of considering only a subset of previous observations when making decisions. Here the case where all previous observations and decisions are considered is an extreme case corresponding to an influence diagram, and the case where only the most recent observations are considered is the opposite extreme corresponding to a Markov decision process. Due to the embedding of the LIMIDs in a Markov decision process (the founder) we also ensure that we are dealing with a true dynamic model that also supports optimization under infinite time horizon.

The example, on the other hand, only illustrates a minor part of the potential of Markov LIMID Processes. In the robot example, for instance, the same LIMID is used in all founder states and under all founder decisions. In some cases it would be more natural to use separate LIMIDs for each combination of founder state and founder action. It is also possible to mix models so that in some founder states or actions a LIMID is used while in others an ordinary Markov decision process is applied.

\section{References}

Cowell, R. G., Dawid, A. P., Lauritzen, S. L., Spiegelhalter, D. J., 1999. Probabilistic Networks and Expert Systems. Springer, New York.

Houben, E., Huirne, R., Dijkhuizen, A., Kristensen, A. R., 1994. Optimal replacement of mastitis cows determined by a hierarchic markov process. Journal of Dairy Science 77, 29752993.

Howard, R., Matheson, J., 1984. Influence Diagrams. Readings in the Principles and Applications of Decision Analysis. Strategic Decision Group, Menlo Park, CA.

Jensen, F. V., 2001. Bayesian Networks and Decision Graphs. Statistics for Engineering and Information Science. Springer-Verlag, New York.

Kaelbling, L. P., Littman, M. L., Cassandra, A. R., 1998. Planning and acting in partially observable stochastic domains. Artificial Intelligence 101 (1-2), 99-134.

Kristensen, A. R., 1988. Hierarchic markov processes and their applications in replacement models. European Journal of Operational Research 35, 207-215.

Kristensen, A. R., 1993. Bayesian updating in hierarchic markov processes applied to the animal replacement problem. European Review of Agricultural Economics 20, 223-239.

Kristensen, A. R., Jørgensen, E., 2000. Multi-level hierarchic markov processes as a framework for herd management support. Annals of Operations Research. 94, 69-89.

Kristensen, A. R., Lien, G., 2002. Optimal length of leys in an area with winter damage problems. In: First European Workshop on Sequential Decisions under Uncertainty in Agriculture and Natural Resources. INRA, September 19-20, 2002, Toulouse, France. 
Lauritzen, S. L., Nilsson, D., 2001. Representing and Solving Decision Problems with Limited Information. Management Science 47, 1235-51.

Lovejoy, W. S., Apr. 1991. A survey of algorithmic methods for partially observed Markov decision processes. Annals of Operations Research 28 (1-4), 47-66.

Mourits, M., Huirne, R., Dijkhuizen, A., Kristensen, A. R., Galligan, D., 1999. Economic optimization of dairy heifer management decisions. Agricultural Systems 61, 17-31.

Oliver, R., Smith, J., 1990. Influence Diagrams, Belief Nets and Decision Analysis. John Wiley and Sons, Chichester, United Kingdom.

Shachter, R., 1986. Evaluating influence diagrams. Operations Research 34, 871-882.

Søllested, T. A., Kristensen, 2002. A sow replacement model using bayesian updating in a 3-level hierarchic markov process. In: First European Workshop on Sequential Decisions under Uncertainty in Agriculture and Natural Resources. INRA, September 19-20, 2002, Toulouse, France.

Smith, J., 1989. Influence diagrams for bayesian decision analysis. European Journal of Operational Research 40, 364-376.

Verstegen, J. A., Sonnemans, J., Huirne, R. B., Dijkhuizen, A. A., Cox, J. C., 1998. Quantifying the effects of sow-herd management information systems on farmers' decision making using experimental economics. American Journal of Agricultural Economics 80, 821. 